\title{
Experimental and Numerical Investigation of the Effect of Process Conditions on Residual Wall Thickness and Cooling and Surface Characteristics of Water-Assisted Injection Molded Hollow Products
}

\author{
Hyungpil Park, ${ }^{1}$ Baeg-Soon Cha, ${ }^{2}$ and Byungohk Rhee ${ }^{3}$ \\ ${ }^{1}$ Mold Technology Team, Hyundai Mobis, 460-30 Sam-dong, Uiwang-si, Gyeonggi-do 437-040, Republic of Korea \\ ${ }^{2}$ Molding \& Forming Technology R\&D Group, Korea Institute of Industrial Technology, 7-46 Songdo-dong, Yeonsu-gu, \\ Incheon 406-840, Republic of Korea \\ ${ }^{3}$ Department of Mechanical Engineering, Ajou University, San 5 Woncheon-dong, Yeongtong-gu, Suwon, \\ Gyeonggi-do 443-749, Republic of Korea \\ Correspondence should be addressed to Hyungpil Park; php76@kitech.re.kr
}

Received 18 September 2014; Revised 17 February 2015; Accepted 18 February 2015

Academic Editor: Aiguo Xu

Copyright (C) 2015 Hyungpil Park et al. This is an open access article distributed under the Creative Commons Attribution License, which permits unrestricted use, distribution, and reproduction in any medium, provided the original work is properly cited.

\begin{abstract}
Recently, water-assisted injection molding was employed in the automobile industry to manufacture three-dimensional hollow tube-type products with functionalities. However, process optimization is difficult in the case of water-assisted injection molding because of the various rheological interactions between the injected water and the polymer. In this study, the boiling phenomenon that occurs because of the high melt temperature when injecting water and the molding characteristics of the hollow section during the water-assisted injection process were analyzed by a water-assisted injection molding analysis. In addition, the changes in the residual wall thickness accompanying changes in the process conditions were compared with the analysis results by considering water-assisted injection molding based on gas-assisted injection molding. Furthermore, by comparing the cooling characteristics and inner wall surface qualities corresponding to the formation of the hollow section by gas and water injections, a water-assisted injection molding technique was proposed for manufacturing hollow products with functionality.
\end{abstract}

\section{Introduction}

Gas-assisted injection molding (GAIM) is the major injection molding technique used to reduce the thickness of thickwalled products $[1,2]$. For this purpose, some researchers applied GAIM to the manufacture of automobile oil and coolant tubes by implementing a complex process (extrusionwelding), such as the process adopted to make metallic pipes. However, the manufacturing process failed because of the formation of irregular hollows and interior surface defects (air bubble) caused by the action of the compressible gas used in the process.

Lately, water-assisted injection molding (WAIM), in which water is used instead of gas, is being applied especially in the automobile industry to achieve high productivity, cost reduction, and lightening of functional hollow products [3]. Water has an excellent effect in terms of enhancing the productivity by reducing the cooling time by $30-40 \%$; this is because water has an excellent cooling characteristic, with its thermal conductivity and heat capacity being 40 and 4 times, respectively, those of a gas. It has an advantage over GAIM in that the hollow products with functionality obtained by WAIM have a thin and fine interior surface and the residual wall thickness is regular unlike in the case of GAIM products $[4,5]$.

However, in WAIM, unstable flow is generated when the water passes through nozzle, and boiling effect is caused when the water is injected into high temperature molten plastic. The 
flow instability and boiling effect of the water cause several defects such as nonuniform RWT, void in the wall, fingering, and double wall which can decrease the strength and stiffness of final products [6].

Yang and Chou and Liu et al. applied GAIM and WAIM in the forming of curve-shaped hollow products and evaluated nonuniform RWT through experiment $[7,8]$. They proposed that the difference in growth speed of frozen layer affected the nonuniform RWT. Liu et al. analyzed penetration length of water for the resin in which glass fiber was added and proposed the changes in RWT affected by viscosity and shrinkage of resin [9]. In particular, the effect of crystallization of RWT that appears due to rapid cooling of resin by high heat capacity of water was examined through experiment [10]. Liu and Lin have analyzed fingering effect in the formed product having rib shape according to process condition in WAIM [11]. However, most of researches were conducted by experiments for process conditions are extremely difficult. This is because the hollow shape formed by water injection is sensitive to changes in the viscosity of the polymer, product shape, and molding process.

In this research, the residual wall thickness distribution of the hollow section created by the passage of water and the molding characteristics were studied by performing a WAIM analysis of the filling behavior of the water that forms the hollow. A GAIM-based WAIM system was developed to test the WAIM; the WAIM analysis results and the residual wall thickness distribution of the hollow formed in accordance with the process conditions were compared and analyzed. In addition, the cooling characteristics and interior surface qualities of GAIM and WAIM products were compared to analyze the effectiveness of WAIM in molding hollows with functionality.

\section{Water-Assisted Injection Molding Analysis}

2.1. Governing Equation. Moldex3D of CoreTech Inc. is the only commercial injection molding software for the WAIM analysis. It was base governing equation of gas-assisted injection molding. The injected fluid applied water instead of gas. The viscosity of the resin at the flow front boundary, which is in contact with water, changes because the boundary is cooled by the water injected into the polymer melt for forming a hollow. It affects the residual wall thickness distribution. Hence, the simulation analysis is necessary for predicting water's filling behavior, hollow residual wall thickness distribution, and the shrinkage distribution in WAIM [12].

In WAIM analysis, the core area where water flows is assumed to have constant pressure. The polymer melt filling process is defined as the process of introducing a nonisothermal, incompressible, and non-Newtonian fluid. Water is injected at a low pressure for forming a hollow, and, hence, it is defined as an incompressible Newtonian fluid. The surface tension at the flow front of the water is not considered. The mass, momentum, and energy conservation equations of the three-dimensional transient nonisothermal process for WAIM analysis are as follows. Here, $p$ represents pressure; $T$, temperature; $u$, velocity; $\tau$, stress tensor; $\rho$, density; $\eta$, viscosity; $k$, thermal conductivity; $C_{p}$, specific heat; and $\dot{\gamma}$, shear strain:

$$
\begin{gathered}
\frac{\partial P}{\partial t}+\nabla \cdot \rho u=0 \\
\frac{\partial}{\partial t}(\rho u)+\nabla \cdot(\rho u u+\tau)=-\nabla p+\rho g \\
\rho C_{p}\left(\frac{\partial T}{\partial t}+u \cdot \nabla T\right)=\nabla(k \nabla T)+\eta \dot{\gamma}^{2}, \\
\tau=-\eta\left(\nabla u+\nabla u^{T}\right) .
\end{gathered}
$$

Viscosity is expressed using the modified Cross model. Here, $\eta_{0}$ is the Newtonian viscosity at zero shear rate, $\tau^{*}$ is the shear stress, and $n$ is the power function exponent:

$$
\begin{gathered}
\eta(T, \dot{\gamma})=\frac{\eta_{0}(T)}{1+\left(\eta_{0} \dot{\gamma} / \tau^{*}\right)^{1-n}}, \\
\eta_{0}(T)=B \operatorname{Exp}\left(\frac{T_{b}}{T}\right) .
\end{gathered}
$$

Further, the flow front boundary location of water injected into the core at a specific time is defined through the pseudoconcentration method. The resin ratio that already occupies the entire volume is defined as parameter $F_{i}$. The inflow amount is calculated by computing the pressure field. Thus, a new $F_{i}$ value is calculated after time $\Delta t$ has passed. Here, only polymer melt exists when $i=1$. If $i$ lies between 0 and 1 , the polymer melt and fluid exist together. When $i=0$, an empty space exists because the resin has not been filled, as shown in

$$
\frac{\left(\partial F_{i}\right)}{\partial t}+\nabla \cdot\left(u F_{i}\right)=0
$$

2.2. Simulation Analysis Definition. The viscosity of the resin at the flow front boundary, which is in contact with water, changes because the boundary is cooled by the water injected into the polymer melt for forming a hollow. It affects the residual wall thickness distribution. Hence, the simulation analysis is necessary for predicting water's filling behavior, hollow residual wall thickness distribution, and the shrinkage distribution in WAIM.

Moldex3D R11.0 of CoreTech Inc. was used for the WAIM analysis. Figure 1 shows the three-dimensional (3D) model of the hollow section molding used for analysis and the 3D finite element model generated for the WAIM analysis. The number of nodes in the finite element model is 377,$933 ; 1,540,882$ elements were generated. The applied resin is GS Caltex's M540 PP (polypropylene: semicrystalline resins). Table 1 lists the process conditions applied in the analysis.

2.3. Analysis Result. Figure 2 shows the filling process of the WAIM. When initially injecting the polymer melt, flow stagnation occurred at a thin functionality part. A short shot occurred even after hollow formation by water injection was completed. Generally, the water injection process in WAIM is 


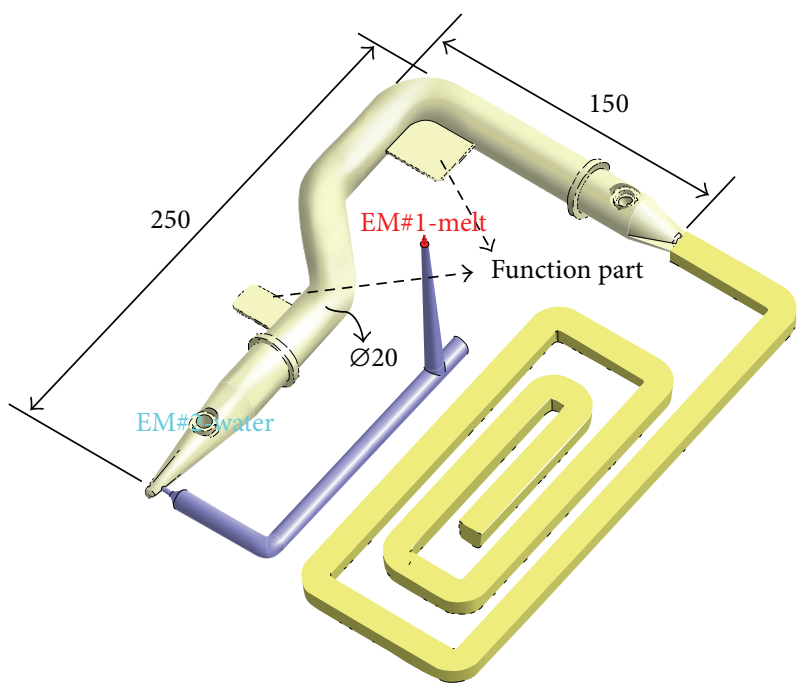

(a)

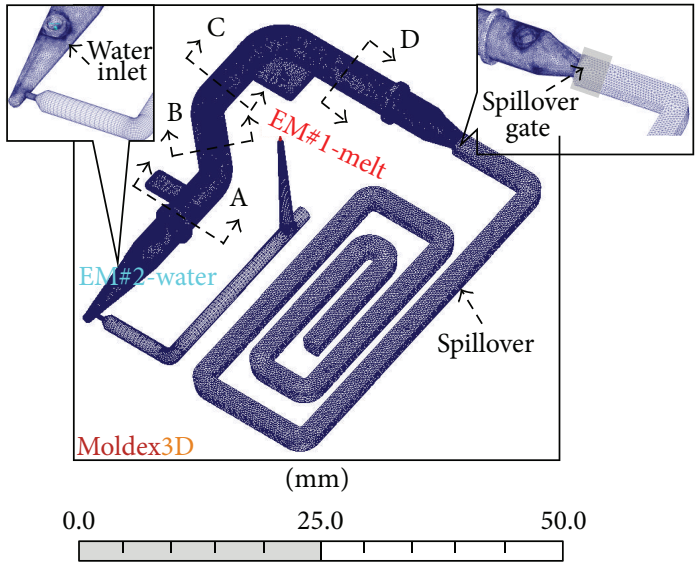

(b)

FIGURE 1: Images of (a) the 3D model and (b) the finite element model for WAIM simulation analysis.

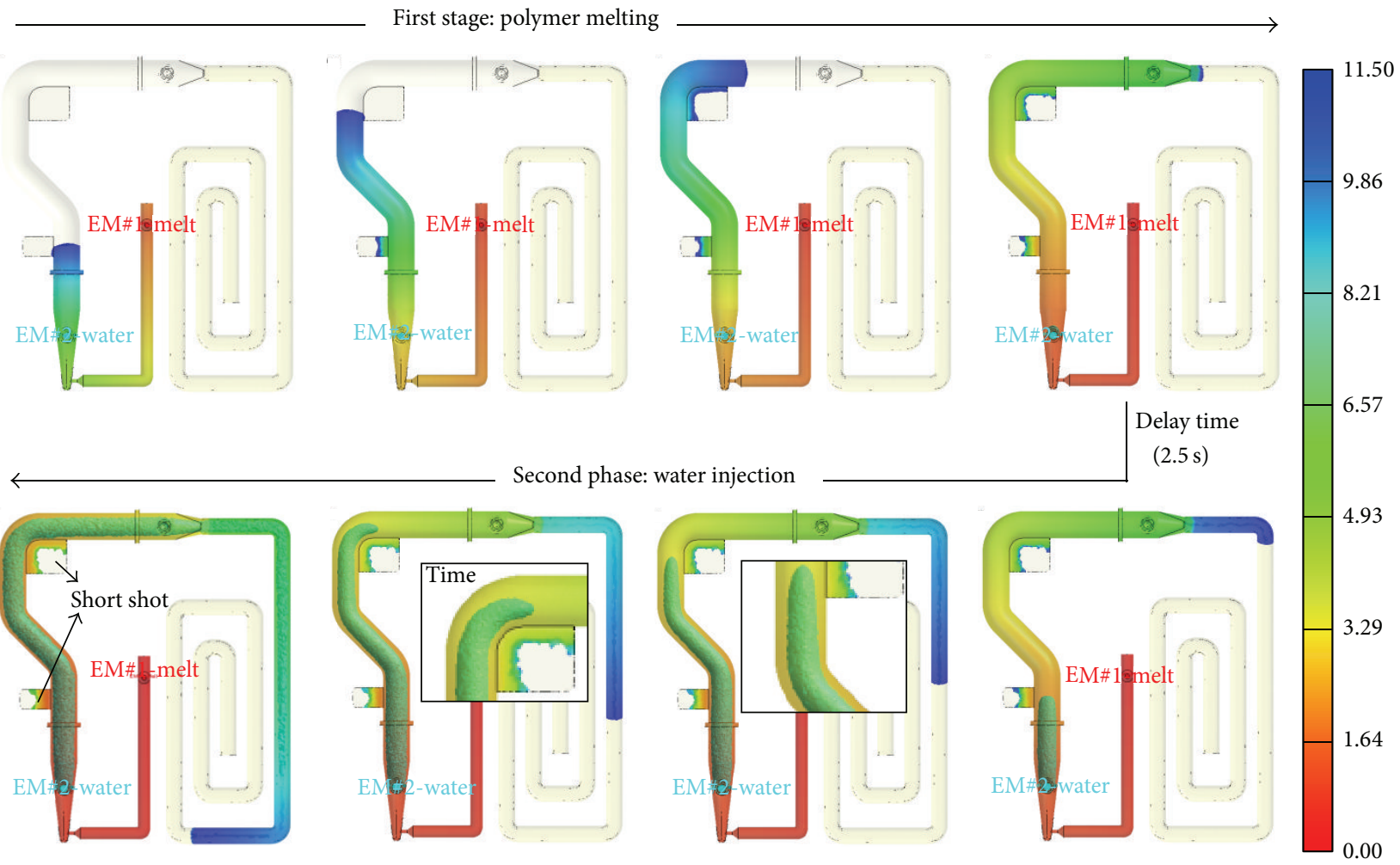

FIGURE 2: Filling patterns for WAIM in CAE analysis.

similar to the process of packing in general injection molding. However, in the case of a thin functionality part, it is difficult to mold by applying water pressure. The packing pressure is applied to fill the functionality part. On the other hand, this is caused by increasing in residual wall thickness due to mold cooling. Therefore, the packing process was excluded from this study to assess the effect of water injection delay time in the WAIM process.
The polymer melt filled in the mold undergoes cooling process during the water injection delay time of $2.5 \mathrm{~s}$. The surface of the melt resin that is in contact with the mold forms a frozen layer during this delay time. The polymer melt of the core layer is maintained in a melting stage, because the frozen layer formed in the surface layer acts as a heat insulator. After the water injection delay time, the high pressure water pushes the polymer melt in the core layer to the spillover cavity and 
TABLE 1: Process conditions for WAIM simulation analysis.

\begin{tabular}{lcc}
\hline Parameter & Unit & PP [M540] \\
\hline Filling time & $\mathrm{S}$ & 3 \\
Melt temperature & ${ }^{\circ} \mathrm{C}$ & 230 \\
Mold temperature & ${ }^{\circ} \mathrm{C}$ & 45 \\
Delay time & $\mathrm{S}$ & 2.5 \\
Water pressure & $\mathrm{MPa}$ & 4.5 \\
Water duration & $\mathrm{S}$ & 6 \\
Water temperature & ${ }^{\circ} \mathrm{C}$ & 25 \\
\hline
\end{tabular}

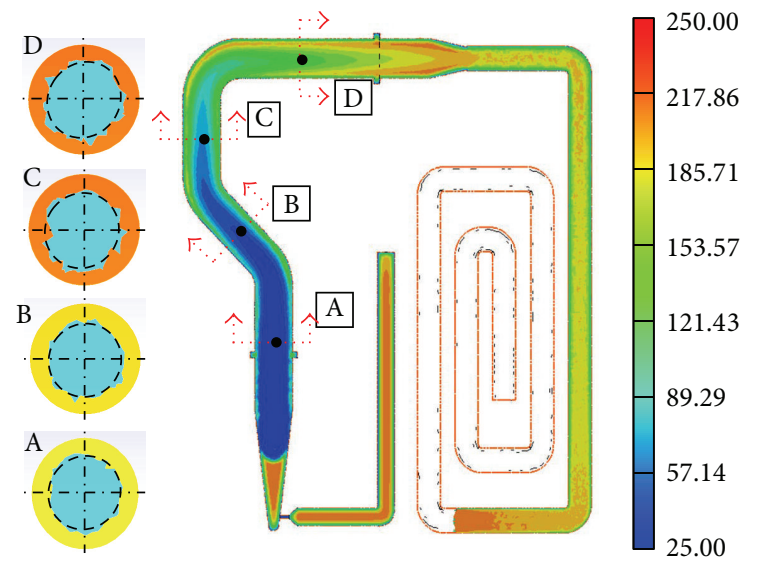

FIGURE 3: The hollow section and temperature distribution after water penetration in simulation analysis.

forms a hollow. At this time, water passing through the curved regions flows along the maximum stream line where flow resistance and pressure are minimum. Hence, the water flows slanted to the inside, leading to a difference between the inner and outer residual walls [13].

Figure 3 shows the hollow section and the temperature distribution of the hollow section. The water inlet where room temperature water is continuously injected for forming the hollow had a low temperature distribution. However, the injected water pushed the high temperature polymer melt as it neared the terminal part of a hollow and received high heat continuously. At a terminal of the hollow, a high temperature of over $150^{\circ} \mathrm{C}$ was formed.

Figure 4 shows the changes in temperature and pressure at locations from A to D. Rapid pressure changes occurred owing to the changes in the initial flow that occurred when injecting water. Thereafter, the pressure gradually increased with the flow resistance when the polymer melt was pushed out to spillover during water injection.

Location A showed a rapid decrease in temperature when water at room temperature was injected to form hollow sections. However, owing to the high temperature polymer melt, the water temperature increased as it neared locations $\mathrm{B}, \mathrm{C}$, and D. In fact, the water temperature was over $100^{\circ} \mathrm{C}$ at $\mathrm{C}$ and $\mathrm{D}$.

Usually, a phase change occurs when water temperature exceeds $100^{\circ} \mathrm{C}$. Density change leads to a large volume expansion. The vaporized water leads to an increase in flow

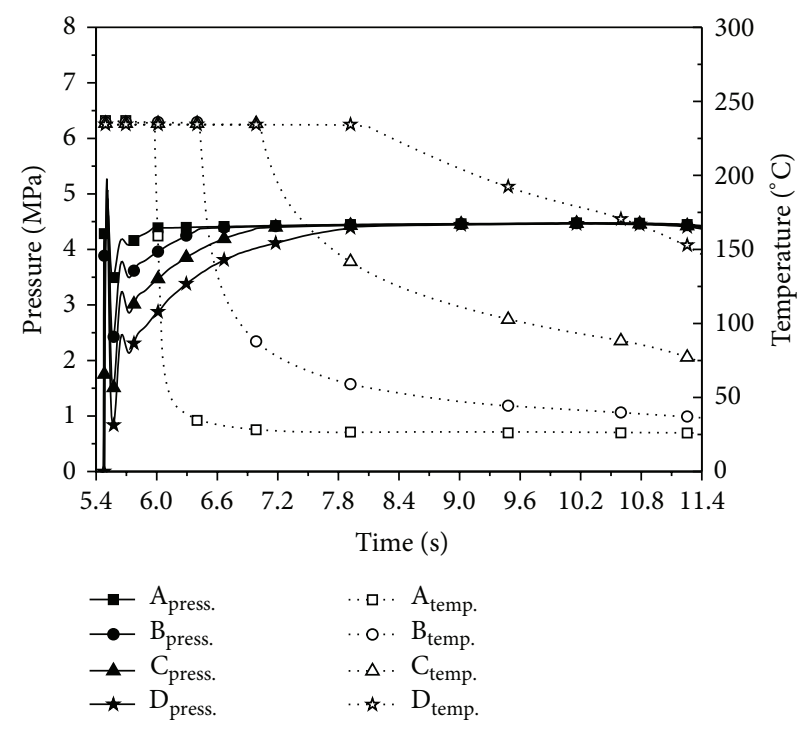

FIgUre 4: Pressure and temperature profiles at the central point of measurement locations (A, B, C, and D).

instability during the hollow formation. Thus, the water injected for forming hollow sections should not undergo any phase changes that are caused by the high temperature of the polymer melt. Table 2 lists the saturated steam table against the temperature and pressure of water [14].

As described in Table 2, a pressure of $3.9 \mathrm{MPa}$ is needed to increase the boiling temperature of water to $250^{\circ} \mathrm{C}$. However, it is difficult to maintain the water pressure during injection, since the volume of the melt resign in the core layer changes as the hollow is formed, and pressure changes owing to the influence of unstable flow when initially injecting water. Further, the specific volume shows a 50 -fold change when the liquid changes to gas at $250^{\circ} \mathrm{C}$. If the pressure at the standard temperature decreases at this time, water would undergo a greater change in specific volume. Accordingly, Polynkin et al. [15] demonstrated that uneven residual wall thickness results from the unstable flow caused by the phase change of water and that the water that penetrates the residual wall vaporizes as it changes phase.

\section{WAIM Experiment}

3.1. GAIM-Based WAIM System. In this study, the GAIMbased WAIM system that injects water using the GAIM system's high pressure gas was applied [16, 17]. Figure 5 shows the lab scale system used in the experiment. The system is composed of three units: the water-providing unit; the measuring process unit, which can control the amount of water injected; and the water injection process unit, which injects water along with high pressure gas to form hollows. Figure 6 shows operating procedure of WAIM using lab scale system.

Figure 7 shows a section of the hollow molded under the experimental conditions listed in Table 1 . The initially formed hollow part was not uniform due to the effect of unstable flow which occurred during the opening of the nozzle for 
TABLE 2: Saturated steam values of water.

\begin{tabular}{lccc}
\hline Temp $\left[{ }^{\circ} \mathrm{C}\right]$ & Pressure $[\mathrm{MPa}]$ & Vapor $\left[\mathrm{m}^{3} / \mathrm{kg}\right]$ & $\mathrm{Liquid}\left[\mathrm{m}^{3} / \mathrm{kg}\right]$ \\
\hline 25 & 0.003 & 43360 & 1.003 \\
100 & 0.101 & 1673 & 1.043 \\
150 & 0.476 & 392.8 & 1.09 \\
200 & 1.554 & 127.4 & 1.156 \\
250 & 3.973 & 50.13 & 1.251 \\
\hline
\end{tabular}

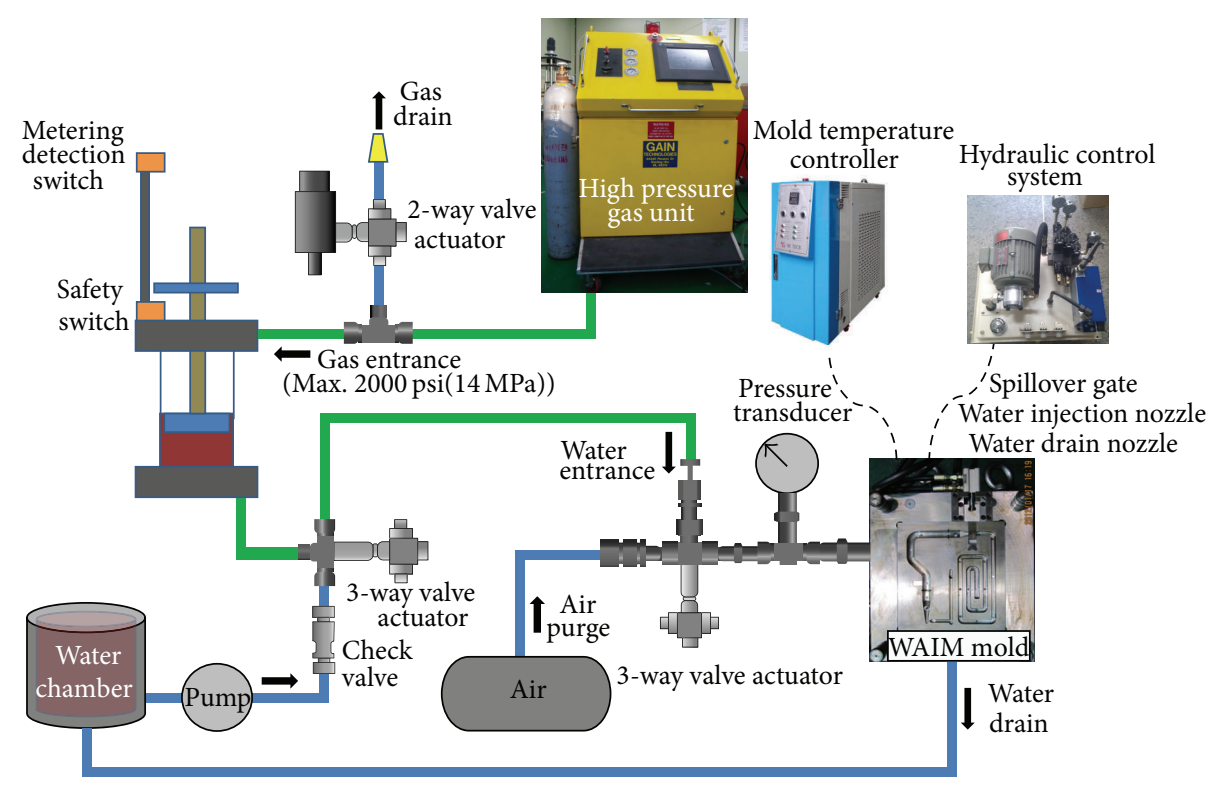

FIgURE 5: Schematic of the GAIM-based WAIM system for experiment.

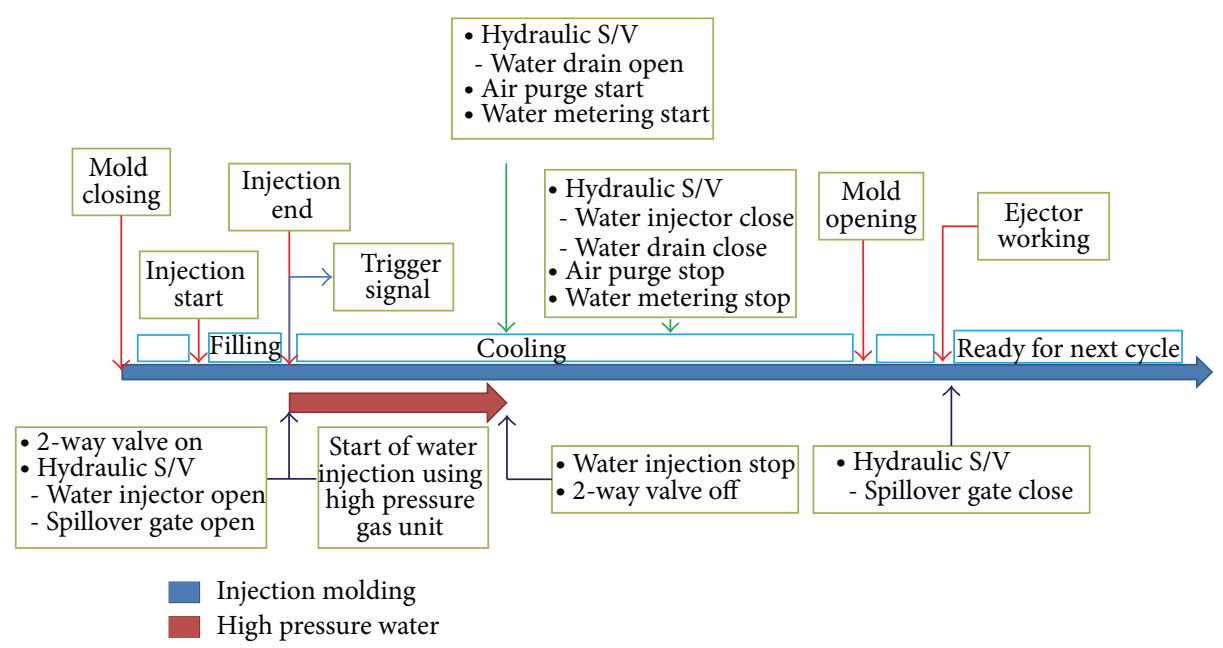

FIGURE 6: Sequence of steps in the GAIM-based WAIM system.

injecting water. After that, a uniform hollow part was formed while flow stabilized. Unstable water flow occurred due to abrupt flow rate and pressure changes while water was passing through the nozzle at the initial stage of water injection, affecting the formation of void in the inner wall. According to research by Polynkin et al. [15], water was atomized through a gap which was formed during the opening of the nozzle.
They suggested that atomized water penetrated the molten resin while forming the hollow product as shown in Figure 8.

Therefore, it was considered that unstable flow and void generated during the initial stage of water injection as in Figure 7 were attributed to these effects.

When the water was injected to form a hollow section, the boundary face of the resin that contacted the flow front of the 


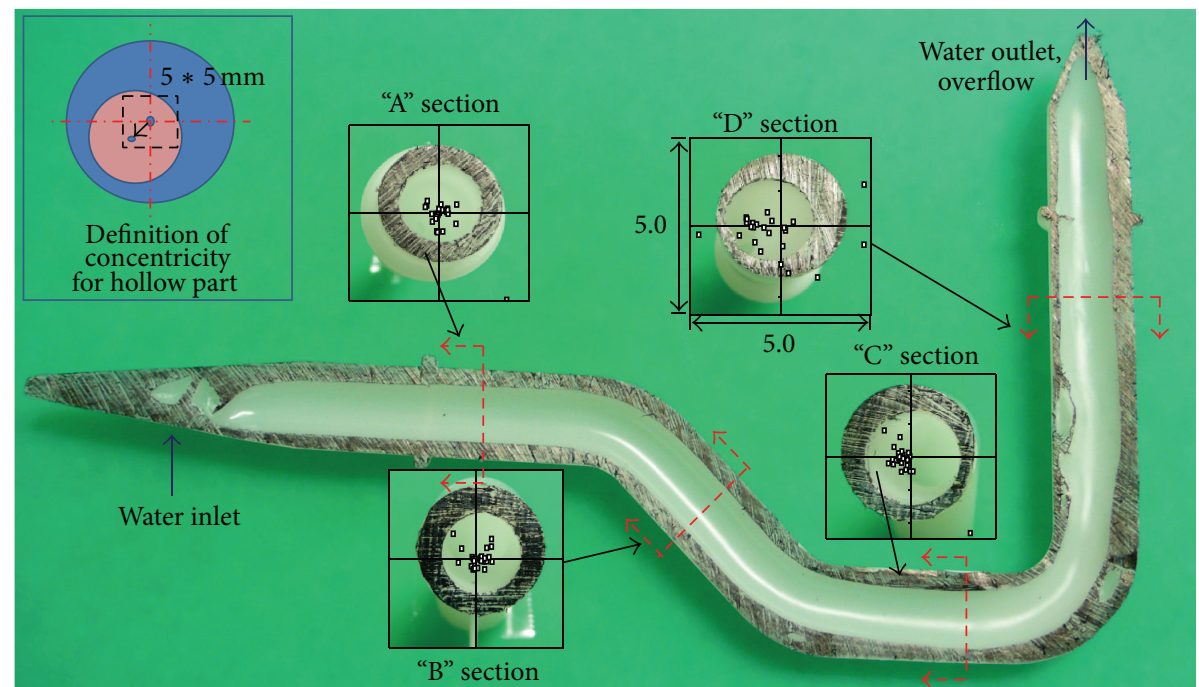

FIGURE 7: Distribution of residual wall thickness and the concentricity of hollow section at measurement locations (A D).

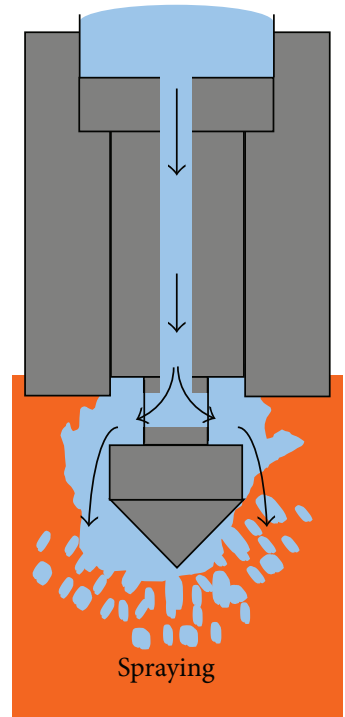

(a)
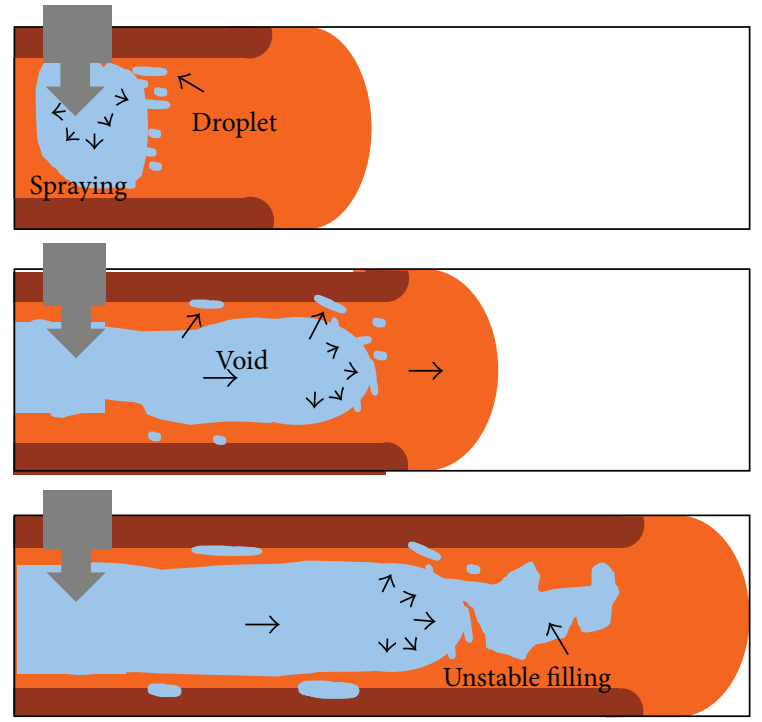

(b)

FIGURE 8: Spraying effect and unstable filling by nozzle design.

water became highly viscous due to abrupt cooling. At this time, elongation and rupture continuously occurred at the cooled boundary face of resin by the injection pressure of the water, thereby forming a hollow product. Meanwhile, boiling occurred at flow front of water by temperature increases since it contacted high temperature resin. This phenomenon was aggregated as it advanced towards the end portion of the hollow product; thus nonuniform hollow section was formed by the unstable flow of vaporized water. It was necessary to increase the boiling point of water by increasing water injection pressure to prevent boiling. However, there was the problem of blow-out, where water penetrated through the flow front of molten resin by a high pressure applied to increase the boiling temperature of water. Therefore, the unstable flow at the end portion of the hollow product was considered as a phenomenon appearing when applied water pressure could not restrict boiling. In this study, the injection pressure of water was set by focusing on the formation of the hollow section rather than on constraining the boiling effect. Owing to the abovementioned effects, the hollow concentricity at location $\mathrm{D}$ was widely distributed.

3.2. Residual Wall Thickness Measurement. Generally, the residual wall thickness of the hollow section formed by WAIM is calculated on the basis of the exterior and interior diameters of the hollow section. However, it is difficult to define the residual wall thickness of locations from $\mathrm{A}$ to $\mathrm{D}$ because the hollow sections formed by water injection have an uneven residual wall thickness in the circumferential direction. As shown in Figure 9, the residual wall thickness 


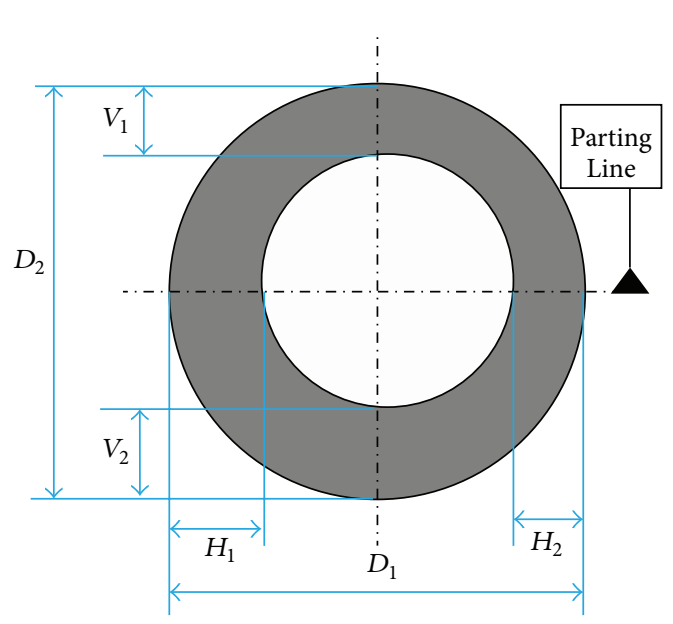

(a)

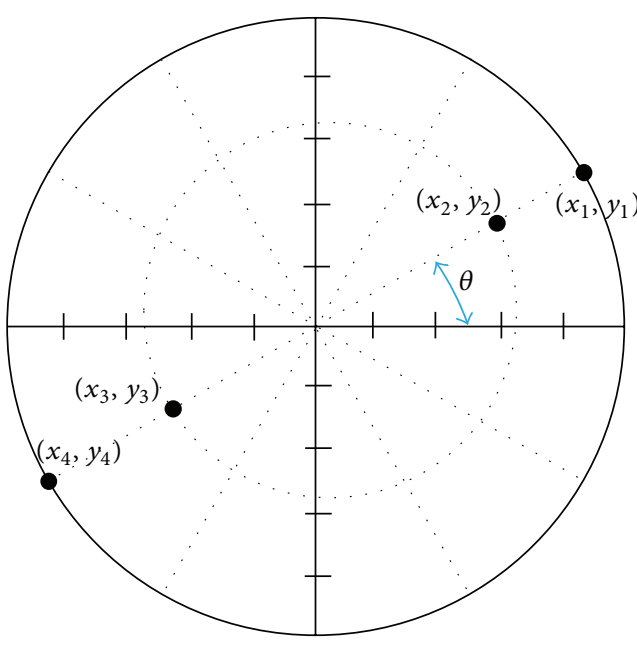

(b)

FIGURE 9: Definition of parameters for (a) the evaluation of residual wall thickness and (b) the calculation of average residual wall thickness using the elliptic equation.

TABLE 3: Experiment design for WAIM.

\begin{tabular}{|c|c|c|c|c|c|}
\hline Case & & Melt temperature $\left[{ }^{\circ} \mathrm{C}\right]$ & Melt temperature $\left[{ }^{\circ} \mathrm{C}\right]$ & Water pressure $[\mathrm{MPa}]$ & Delay time [s] \\
\hline \multirow{3}{*}{ Melt temperature $\left[{ }^{\circ} \mathrm{C}\right]$} & Low & 210 & & & \\
\hline & Medium & 230 & 45 & 4.5 & 2.5 \\
\hline & High & 250 & & & \\
\hline \multirow{3}{*}{ Melt temperature $\left[{ }^{\circ} \mathrm{C}\right]$} & Low & & 35 & & \\
\hline & Medium & 230 & 45 & 4.5 & 2.5 \\
\hline & High & & 55 & & \\
\hline \multirow{3}{*}{ Water pressure $[\mathrm{MPa}]$} & Low & & & 3.5 & \\
\hline & Medium & 230 & 45 & 4.5 & 2.5 \\
\hline & High & & & 5.5 & \\
\hline \multirow{3}{*}{ Delay time $[\mathrm{s}]$} & Low & & & & 0.5 \\
\hline & Medium & 230 & 45 & 4.5 & 2.5 \\
\hline & High & & & & 4.5 \\
\hline
\end{tabular}

of a hollow section towards the horizontal and vertical directions from Parting Line $(\mathrm{P} / \mathrm{L})$ was measured for comparing the residual wall thicknesses obtained from the simulation analysis with the actual values from the WAIM experiment. After that, the measurement value was approximated as inner and outer side ellipses that formed a hollow section. The residual wall thickness was calculated at $30^{\circ}$ intervals from the center of the exterior ellipse by using (1). Five samples were molded under each experimental condition. The residual wall thicknesses measured at locations from $\mathrm{A}$ to $\mathrm{D}$ were averaged. The experiment and analysis results were compared:

$$
\|\delta\|=\sqrt{\left(x_{i+1}-x_{i}\right)^{2}+\left(y_{i+1}-y_{i}\right)^{2}} .
$$

3.3. Effects of the Process Conditions of GAIM-Based WAIM on Residual Wall Thickness. The effects of major WAIM process parameters, resin temperature, mold temperature, water pressure, and water injection delay time, on the residual wall thickness during the formation of the hollow section were analyzed. Table 3 lists the experiment conditions. Figures 10 13 show the measured residual wall thicknesses of the hollow products for experiment and simulation analysis.

Figure 10 shows the changes in the residual wall thickness of the hollow section obtained experimentally and analytically for different melt temperatures. The residual wall thickness decreased as the resin temperature increased. Generally, the resin viscosity is low at high temperatures, leading to a decrease in the flow resistance during the formation of the hollow section by water injection. Furthermore, the frozen layer formed by the cooling caused when the polymer melt touches the mold wall affects the residual thickness of the hollow section. Thus, changes in resin temperature while the other process conditions are maintained constant affect the growth speed of the frozen layer, consequently affecting the residual wall thickness of the hollow section $[18,19]$.

Owing to cooling, a high viscosity film is formed at the polymer melt boundary that encounters water during the formation of the hollow section. This film stretches and fractures because of the pressure of the injected water 


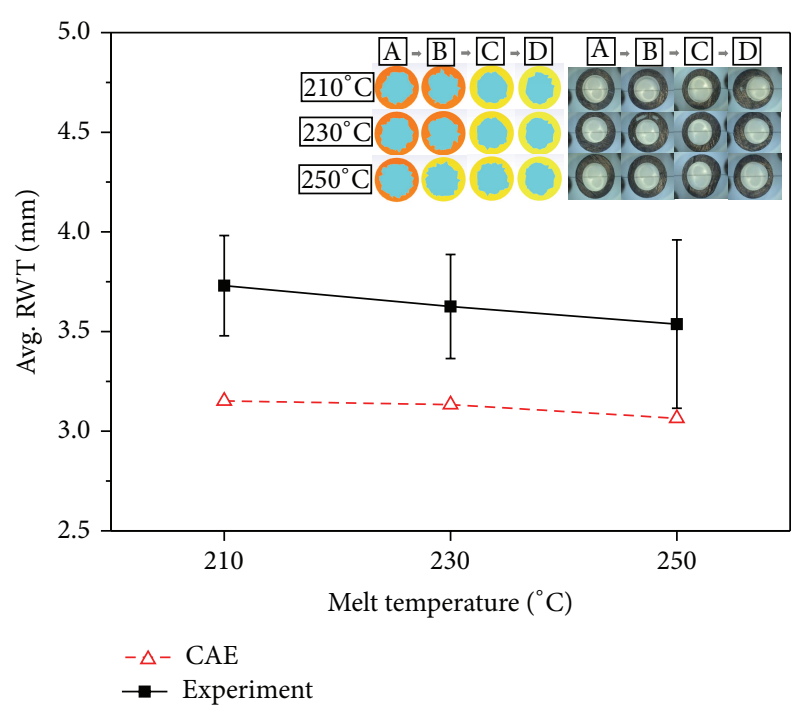

FigURE 10: Effect of melt temperature on average residual wall thickness for the experiment and analysis results.

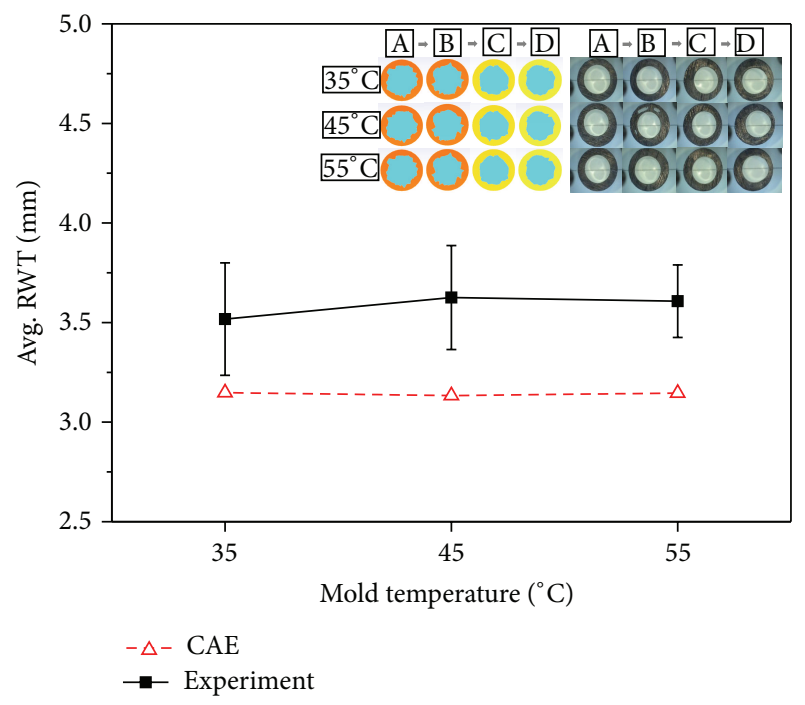

FIGURE 11: Effect of mold temperature on average residual wall thickness for the experiment and analysis results.

and forms a hollow section. At higher melt temperatures, thinner high viscosity films are formed; thus, the residual wall thickness of the hollow section formed by the pressure of the injected water is sensitive to the melt temperature. Further, as seen from the analysis results, at high melt temperatures, the effect of boiling of water becomes more pronounced, leading to the formation of a hollow section with uneven residual wall thickness. The wide distribution of the residual wall thickness is a result of these effects. The experiment and analysis results showed similar tendencies in the changes in residual wall thickness, but the deviation of experiment residual wall thicknesses from the analysis results was great. This is attributed to the fact that the WAIM analysis did

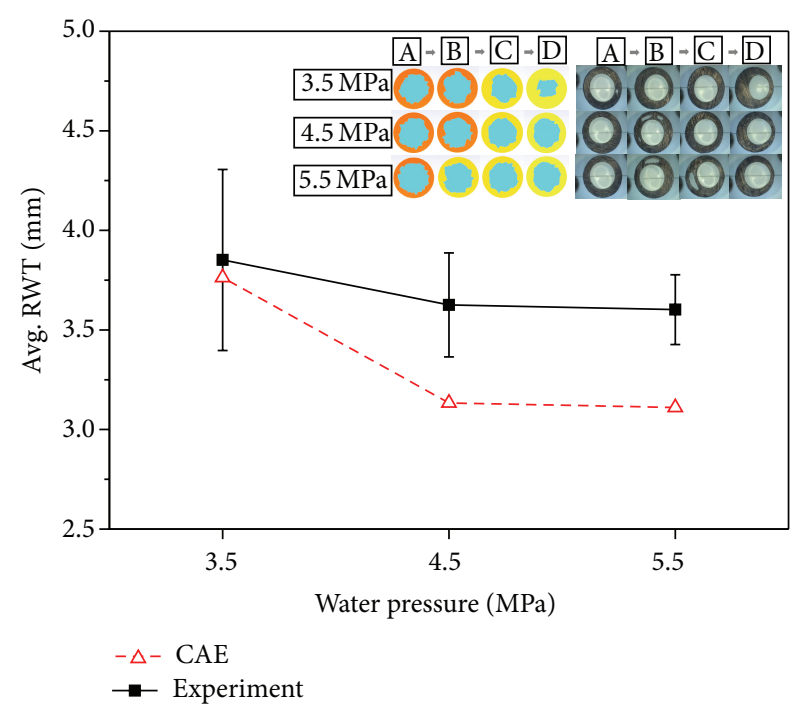

FIGURE 12: Effect of water pressure on average residual wall thickness for the experiment and analysis results.

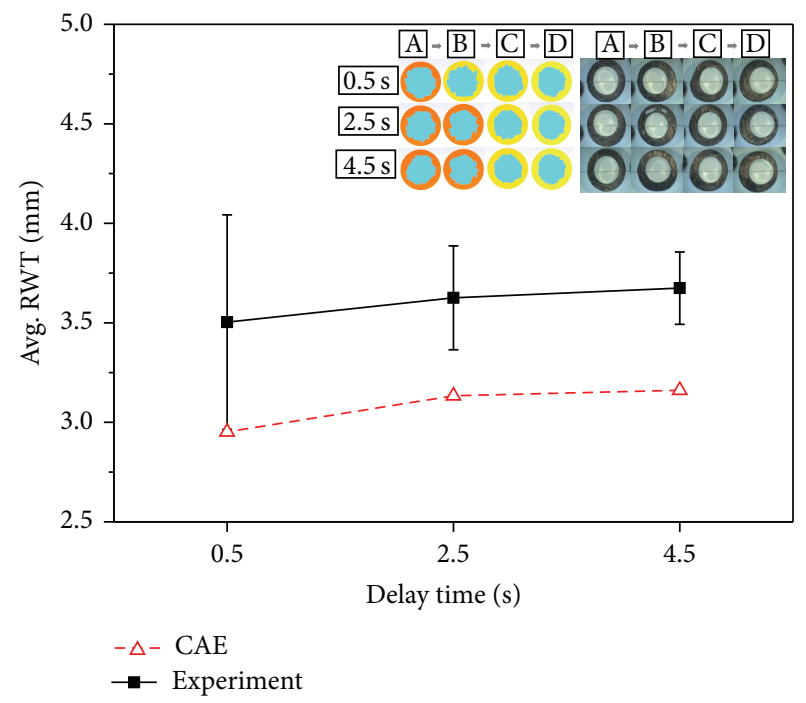

FIGURE 13: Effect of delay time on average residual wall thickness for the experiment and analysis results.

not consider changes in viscosity at the flow front section depending on the injected water.

Figure 11 shows the changes in the residual wall thickness of the hollow section with mold temperature. No large changes were observed in the residual wall thickness in the experiment and analysis results. This is because the frozen layer that formed on the inner surface of the wall during water injection has a greater effect than the mold temperatureinduced frozen layer formed on the outer wall.

Figure 12 shows the changes in the residual wall thickness with water pressure. In WAIM, a hollow section is formed by pushing the polymer melt forward through water injection. Hence, water pressure influences the wall thickness of the hollow section. In fact, the flow speed of the polymer melt 
TABLE 4: Thermal properties of $\mathrm{N}_{2}$ gas and water.

\begin{tabular}{lccc}
\hline & Density $\left[\mathrm{g} / \mathrm{cm}^{3}\right]$ & Specific heat $[\mathrm{J} / \mathrm{kg} \cdot \mathrm{C}]$ & Thermal conductivity $[\mathrm{W} / \mathrm{m} \cdot \mathrm{C}]$ \\
\hline $\mathrm{N}_{2}$ & $1.123 E-03$ & 1041 & 0.025 \\
Water & 0.988 & 4180 & 0.643 \\
\hline
\end{tabular}

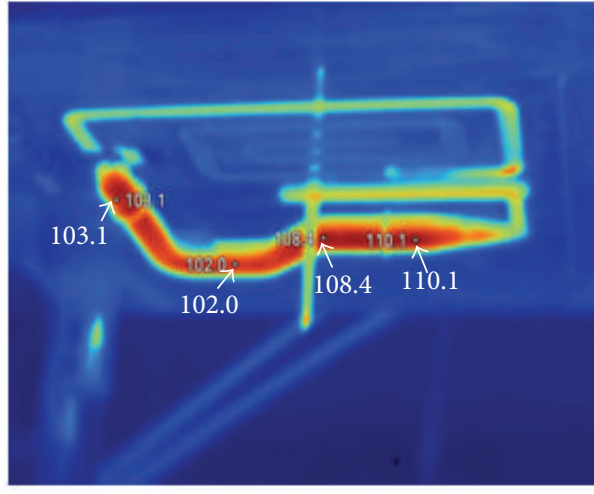

(a)

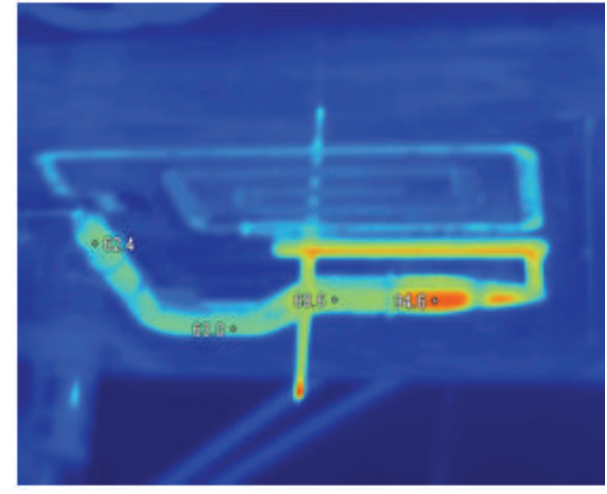

(b)

FIGURE 14: Comparison of the temperature distributions obtained by (a) GAIM and (b) WAIM.

that fills the spillover is determined by the water pressure. If water pressure decreases, the flow speed of the polymer melt that fills with spillover decreases and flow resistance increases because of the increase in viscosity. Thus, if sufficient water pressure to overcome the flow resistance is not applied, the hollow section formed develops a short shot and the residual wall thickness increases. On the contrary, if water pressure is high, the flow resistance decreases because of the viscosity effect of the polymer melt that is filled by spillover. Thus, an increase in water pressure leads to the formation of thinner hollow sections, and deviation in residual wall thickness at the water inlet and terminal section reduces.

After the first polymer melt filling is completed, water injection is carried out after a specific time delay to form the frozen layer of the hollow section. With an increase in the delay time, the frozen layer at the surface where the polymer melt encounters the molding surface grows; thus, the residual wall thickness increases. As shown in Figure 13, both experiment and analysis results showed an increase in the residual wall thickness as the water injection delay time got longer. In addition, the residual wall thickness distribution decreased because the melt temperature of the core layer is uniform over the frozen layer surface.

\section{Comparisons of Gas- and Water-Assisted Injection Molding}

4.1. Cooling Characteristics. In WAIM, water is injected into a high temperature polymer melt to form a hollow section. At the same time, the injected water cools the resin. As shown in Table 4, water has better thermal property than gas and increases the cooling efficiency by more than $30 \%$; thus, the productivity can be increased. Further, water's cooling characteristic affects the crystallization of the semicrystalline resin and induces the formation of a hollow section with characteristic differences in the interior wall surface [20].

An infrared thermal imaging camera (Fluke, Ti50 Thermal Imager) was used for analyzing the cooling characteristics of the molding product made by using gas and water. The surface temperature was measured after opening the molding $20 \mathrm{~s}$ after the completion of the water injection process. As shown in Figure 14, the use of water yields a lower temperature distribution of the molding product than when $\mathrm{N}_{2}$ gas was used. This means that the high specific heat and thermal conductivity of water have a great effect on the molding product cooling, and it can be seen that WAIM is effective for achieving high productivity.

4.2. Interior Wall Surface Characteristics. Figure 15 shows the interior surfaces qualities of the hollow sections formed by applying gas and water. Confocal laser scanning microscopy (Olympus, LEXT OLS 3100) was performed to measure the interior wall surface characteristics. Figure 15(a) shows the case in which gas was applied and spherulite was formed by the vaporization and crystallization in the interior walls of the hollow section. The low specific heat and thermal conductivity of gas slow the formation of the frozen layer in the interior wall of hollow section. Therefore, even though $\mathrm{N}_{2}$ gas injected at a high pressure forms hollow sections, it also penetrates the residual wall. When the interior pressure is removed after the completion of the formation of the hollow section, the gas that penetrated inside the residual wall escapes outside because of the difference in pressure. Minute vapor is formed inside the surface. Furthermore, the slow cooling of the semicrystalline resin polypropylene leads to the formation of a rough inner surface because of the coarsening of the crystal. Figure 15(b) shows the case in which water was applied. Water induces rapid cooling of the inner surface of 

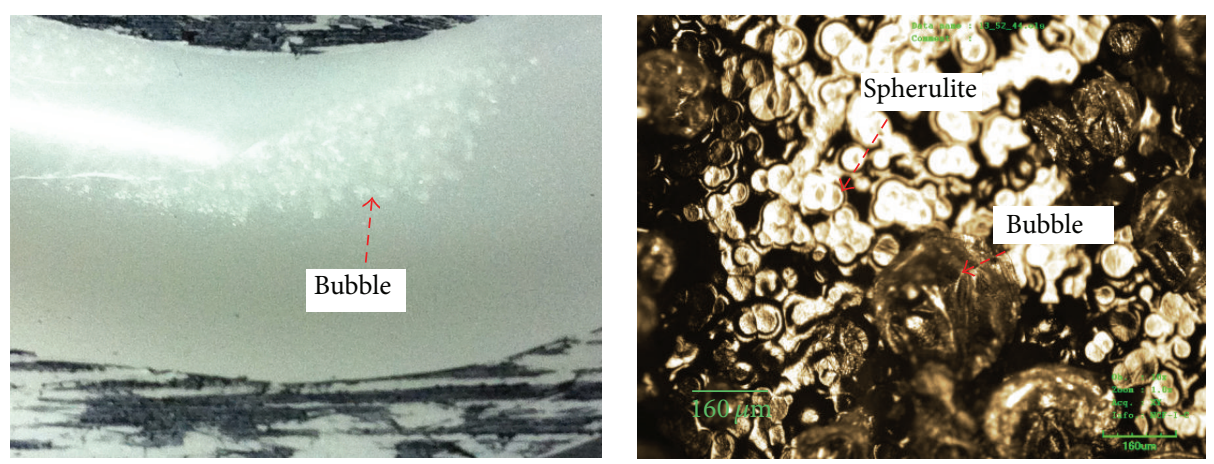

(a)
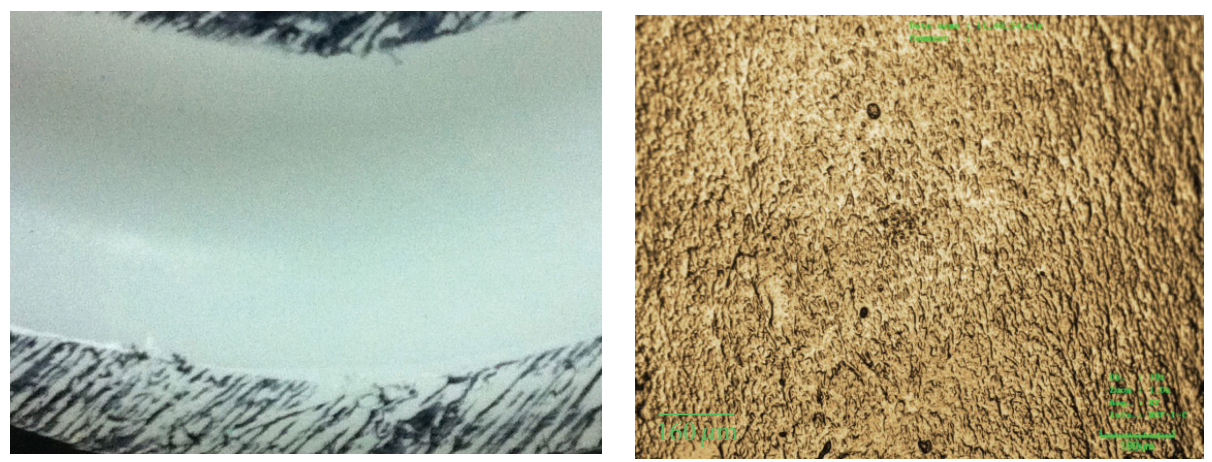

(b)

FIGURE 15: Images obtained by scanning confocal microscopy and the measured inner surface in (a) GAIM and (b) WAIM products.

the hollow section, leading to the formation of fine crystals [21]. The inner surface characteristic is fine.

The roughness of the inner surfaces of the hollow sections formed by GAIM and WAIM was compared. A rough surface was obtained by GAIM, and the average roughness of the inner wall was high $8.875 \mu \mathrm{m}$. On the contrary, a fine inner surface was formed by WAIM, and the average roughness in the interior was $2.843 \mu \mathrm{m}$. Thus, it was verified that WAIM was effective in forming hollow products with functionality.

\section{Conclusions}

In this study, A GAIM-based WAIM system has been developed to molding of three-dimensional hollow products. The developed system was used to analyze the changes in residual wall thickness with process conditions. These results were compared to the WAIM simulation results. The residual wall thickness was mainly influenced by the melt temperatures, water pressure, and water injection delay time. The frozen layer affects residual wall thickness. In particular, the high viscosity film, created by cooling at the polymer melt boundary where the polymer melt encounters water, is stretched and fractured and affects the even distribution of residual wall thickness. The flow characteristics of water were analyzed through a WAIM analysis. The difference between the outer and inner residual wall thicknesses, which is observed at curved regions of hollow sections, is a result of the water flow along the maximum flux line where flow resistance and pressure are minimum. Further, the injected water boils near the terminal part of the hollow section as the temperature increases because of the high temperature polymer melt. The unstable flow resulting from the boiling phenomenon leads to uneven residual wall thicknesses. A comparison of the cooling characteristics and inner wall surface qualities of GAIM and WAIM products reveals that WAIM is effective for forming three-dimensional hollow products with functionalities.

\section{Conflict of Interests}

The authors declare no competing financial interests.

\section{References}

[1] DuPont, "Gas-injection moulding with DuPont engineering polymers," Company Technical Report TRG 3060, DuPont, 1996.

[2] G. Menges, W. Michaeli, and P. Mohren, How to Make Injection Molds, HANSER, New York, NY, USA, 2000.

[3] H. P. Heim and H. Potente, Specialized Molding Techniques, Plastics Design Library, New York, NY, USA, 2001.

[4] W. Michaeli, A. Brunswick, and M. Gruber, "Step on the gas with water injection. Water-assisted injection moulding (WAIM): an alternative to gas injection?" Kunststoffe Plast Europe, vol. 89, no. 4, pp. 20-21, 1999.

[5] W. Michaeli, T. Jüntgen, and A. Brunswick, "WIT-En route to series production: first industrial application of the water injection technique," Kunststoffe Plast Europe, vol. 91, no. 3, pp. 37-39, 2001. 
[6] S. Sannen, J. D. Keyzer, and P. V. Puyvelde, "Formation of part defects in water-assisted injection molding (WAIM): influence of process and material parameters," in Proceedings of the ANTEC, pp. 1557-1564, 2014.

[7] S. Y. Yang and H. L. Chou, "Study on the residual wall thickness at dimensional transitions and curved sections in gas-assisted molded circular tubes," Polymer Engineering \& Science, vol. 42, no. 1, pp. 111-119, 2002.

[8] K.-Y. Lin, F.-A. Chang, and S.-J. Liu, "Using differential mold temperatures to improve the residual wall thickness uniformity around curved sections of fluid assisted injection molded tubes," International Communications in Heat and Mass Transfer, vol. 36, no. 5, pp. 491-497, 2009.

[9] S. J. Liu, M. J. Lin, and Y. C. Wu, "An experimental study of the water-assisted injection molding of glass fiber filled polybutylene-terephthalate (PBT) composites," Composites Science and Technology, vol. 67, no. 7-8, pp. 1415-1424, 2007.

[10] S.-J. Liu and W.-K. Chen, "Experimental investigation and numerical simulation of cooling process in water assisted injection moulded parts," Plastics, Rubber and Composites, vol. 33, no. 6, pp. 260-266, 2004.

[11] S.-J. Liu and S.-P. Lin, "Factors affecting the formation of fingering in water-assisted injection-molded thermoplastics," Advances in Polymer Technology, vol. 25, no. 2, pp. 98-108, 2006.

[12] R.-Y. Chang, C.-T. Huang, W.-H. Yang, M.-H. Tsai, K.-I. Lu, and S.-J. Liu, "The investigation of flow behavior of polymeric melts in the water assisted injection molding," in Proceedings of the Annual Technical Conference (ANTEC '04), pp. 566-569, Chicago, Ill, USA, May 2004.

[13] J.-G. Yang and X.-H. Zhou, "Numerical simulation on residual wall thickness of tubes with dimensional transitions and curved sections in water-assisted injection molding," Journal of Applied Polymer Science, vol. 128, no. 3, pp. 1987-1994, 2013.

[14] http://www.efunda.com.

[15] A. Polynkin, L. Bai, J. F. T. Pittman et al., "Water assisted injection moulding: development of insights and predictive capabilities through experiments on instrumented process in parallel with computer simulations," Plastics, Rubber and Composites, vol. 37, no. 2-4, pp. 131-141, 2008.

[16] H. Zhou, Y. Chen, Z. Zhang, and H. Yang, "Simulation and experiment research on the proportional pressure control of water-assisted injection molding," Chinese Journal of Mechanical Engineering (English Edition), vol. 25, no. 3, pp. 430-438, 2012.

[17] R. Protte, H. Bangert, and C. Cooper, "Water-assist injection molding - an innovative process technology for productivity improvement: developments in processing, equipment and materials," in Proceedings of the Annual Technical Conference (ANTEC '03), pp. 404-408, 2003.

[18] S.-J. Liu and Y.-S. Chen, "Water-assisted injection molding of thermoplastic materials: effects of processing parameters," Polymer Engineering and Science, vol. 43, no. 11, pp. 1806-1817, 2003.

[19] J. G. Yang, X. H. Zhou, and Q. Niu, "Model and simulation of water penetration in water-assisted injection molding," International Journal of Advanced Manufacturing Technology, vol. 67, no. 1-4, pp. 367-375, 2013.

[20] W. Michaeli, A. Brunswick, and C. Kujat, "Reducing cooling time with water-assisted injection moulding," Kunststoffe Plast Europe, vol. 90, no. 8, pp. 25-28, 2000.

[21] B. Wang, H.-X. Huang, and H.-Y. Lu, "Crystal morphology of water-assisted injection molded high-density polyethylene with two different molecular weights," Journal of Macromolecular Science Part B: Physics, vol. 50, no. 8, pp. 1615-1624, 2011. 

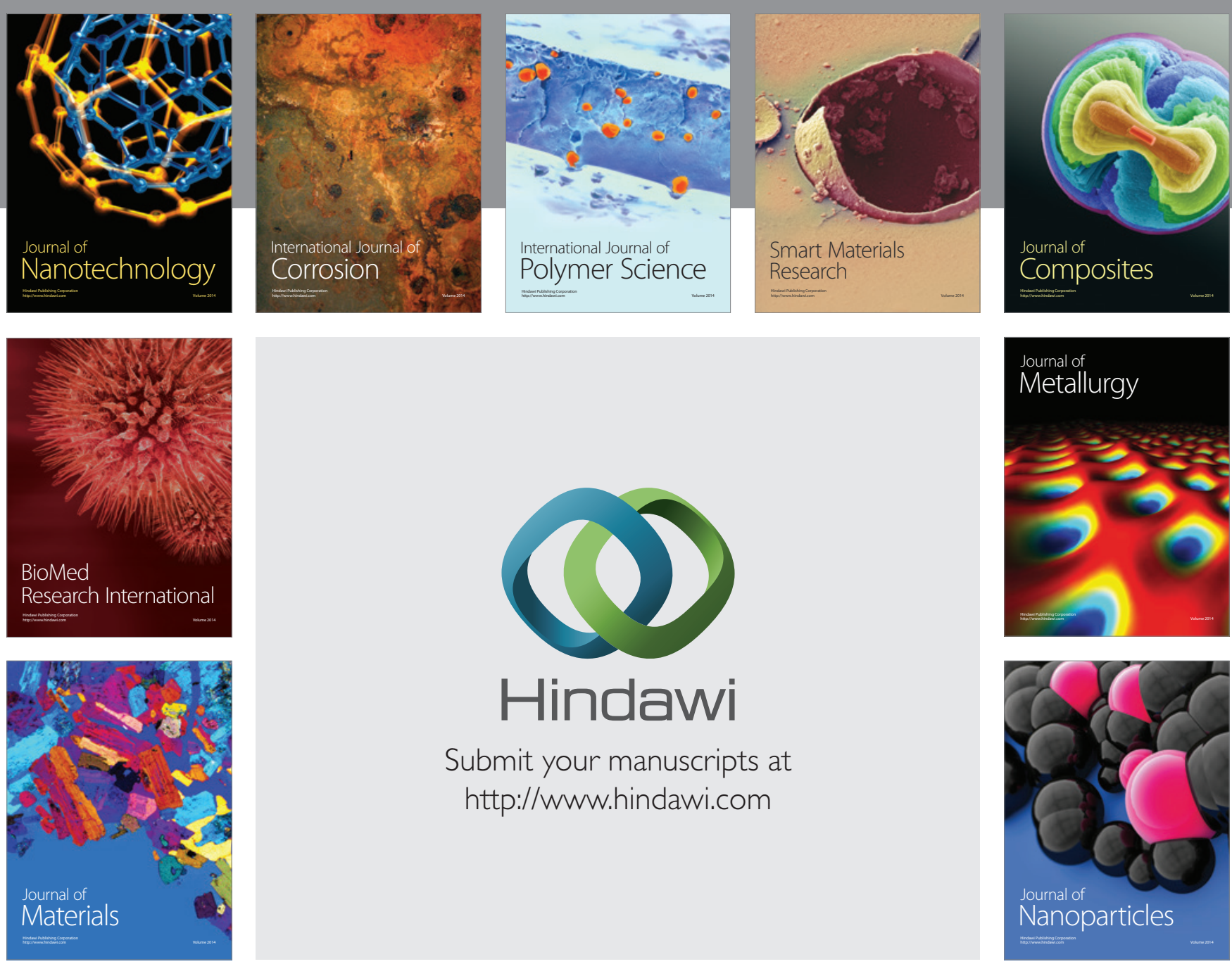

Submit your manuscripts at http://www.hindawi.com
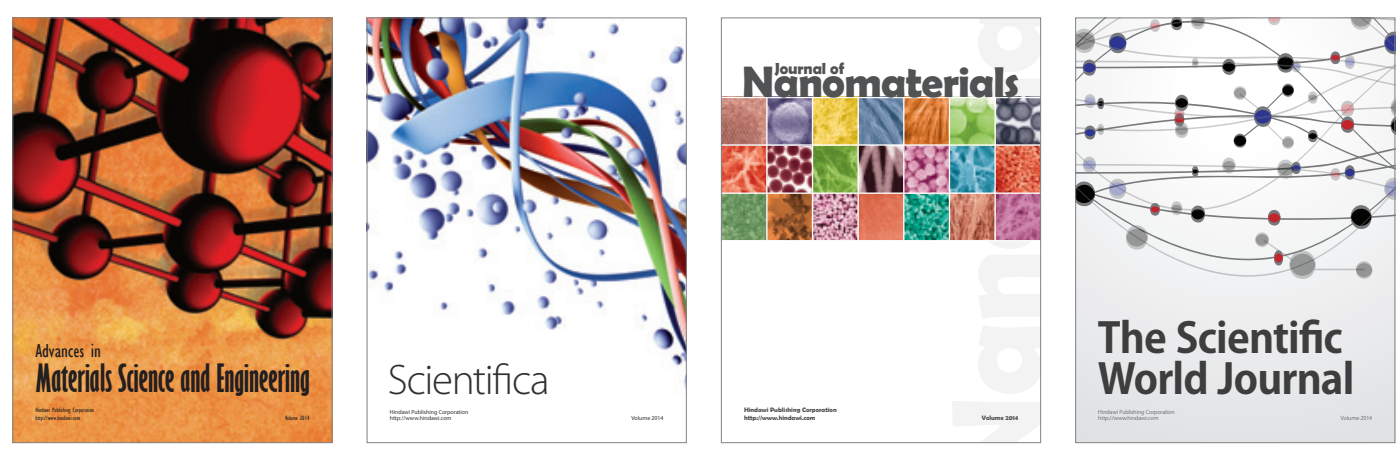

\section{The Scientific World Journal}
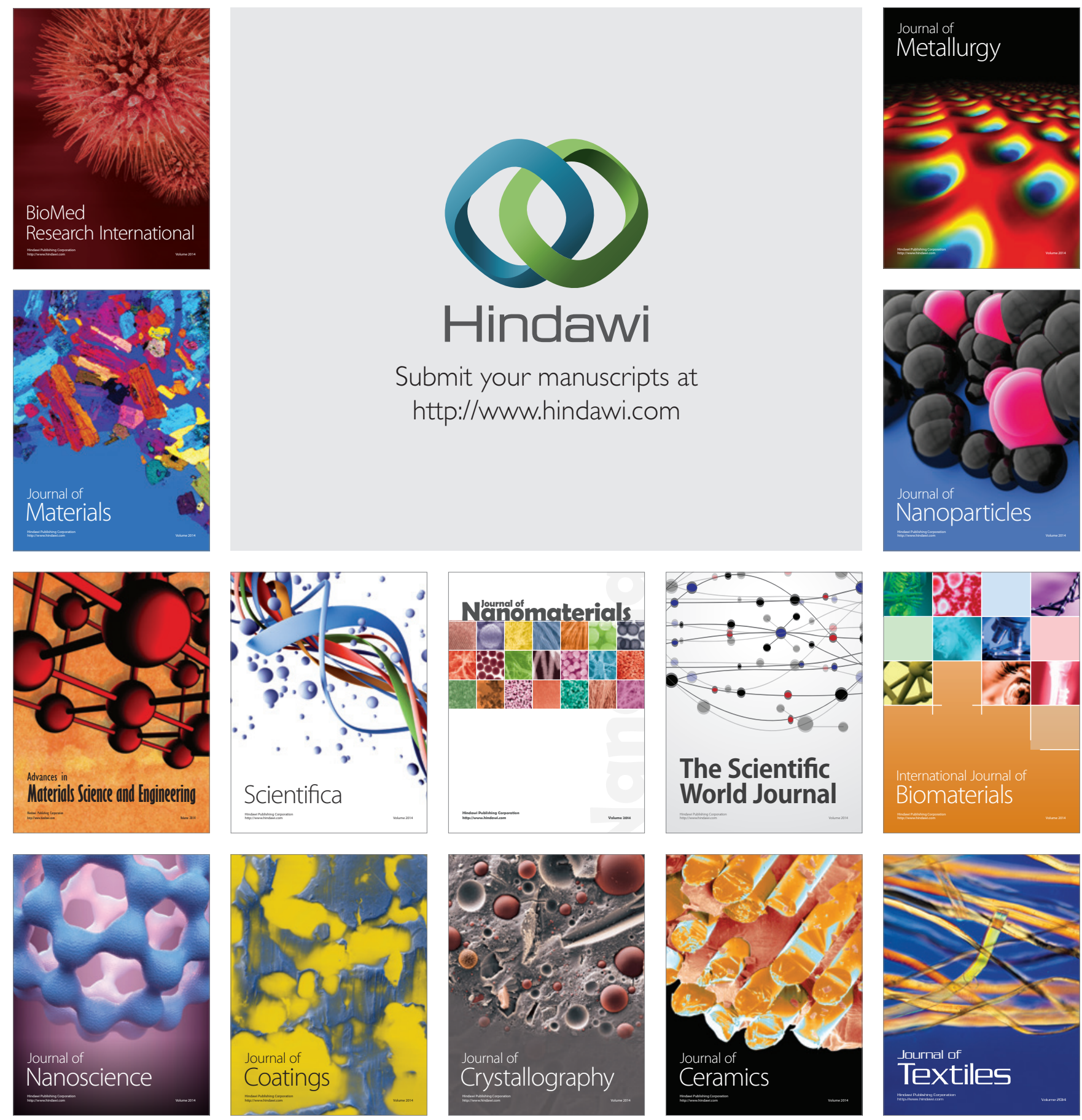